\title{
小鼠肝脏金属硫蛋白消除· $\mathrm{OH}$ 自由基 能力的变化动态 ${ }^{*}$
}

\author{
高崇明张爱琴 \\ (北京大学生物学系, 北京 100871)
}

\author{
杨 立 庄 \\ (北京大学地理学系, 北京 100871)
}

\section{关键词金属硫蛋白、OH 自由基、衰老}

金属硫蛋白 (metallothioneins, MTs) 是普遍存在于生物体中的一种富含半胱氨酸、可 诱导的金属结合蛋白. 近年来, MTs 在生命过程中所起的作用正日益引起人们的重视. 体 外细胞培养实验表明, MTs 具有消除 ${ }^{\circ} \mathrm{OH}$. 自由基的能力 ${ }^{[1]}$. 按照 Denham Harman 所提出 的机体衰老的自由基学说认为, 生物体随龄增大的退行性变化是由于自由基的副作用引起的, 而且自由基促使机体加速衰老的作用也已不断得到证实. 因此, 这就可能意味着 MTs 的生 理功能是与延缓细胞及机体的衰老有一定的关系. 但是, 到目前为止尚未见到有人报道, 在细 胞或机体衰老过程中, MTs 含量水平及消除 ${ }^{\circ} \mathrm{OH}$ 自由基能力的变化动态, 也未见到有人进 行 MT-I 和 $\mathrm{MT}-\mathrm{II}$ 消除 $\cdot \mathrm{OH}$ 自由基能力的比较研究. 我们的研究工作, 主要就是围绕着 这两个问题展开的.

\section{一、材料和方 法}

试验动物为 ICR/JCL 雄性小鼠.

1. MTs 提取取不同日龄的小鼠,断头处死, 取出肝脏, 按 1:1的比例加人 $\mathrm{pH} 8.6$, $0.01 \mathrm{~mol} / \mathrm{L}$ Tris- $\mathrm{HCl}$ 缓冲液, 匀浆. 匀浆液 $24500 \times g, 4^{\circ} \mathrm{C}$ 离心 $10 \mathrm{~min}$. 收集上清液, 在 $100^{\circ} \mathrm{C}$ 水浴上加热 $2 \mathrm{~min}$. 然后, $24500 \times g$ 再离心 $10 \mathrm{~min}$. 所得上清液即是 $\mathrm{MTs}$ 粗提取液, 它 可作为消除 ${ }^{\circ} \mathrm{OH}$ 自由基能力的测定样品.

2. MTs 含罟测定本实验用原子吸收光谱(AAS)的方法测定 MTs 含量.

(1) 样品的处理. 在 $100 \mu 1$ 上述 MTs 粗提取液中,加人 $\mathrm{pH} 8.6,0.01 \mathrm{~mol} / \mathrm{L}$ Tris- $\mathrm{HCl}$ 缓冲液 $600 \mu \mathrm{l}, 100 \mu \mathrm{l}$ 浓度为 $20 \mu \mathrm{g} / \mathrm{ml}$ 的 $\mathrm{CdCl}_{2}$ 溶液，混匀. 然后再加人 $2 \%$ 牛血红蛋白 $100 \mu \mathrm{l}$, 混匀, 静置 $10 \mathrm{~min}$, 沸水浴中加热 $2 \mathrm{~min} .24500 \times \mathrm{g}$ 离心 $10 \mathrm{~min}$, 收集上清液。在此上 清液中再次加人 $2 \%$ 牛血红蛋白 $100 \mu 1$, 混匀, 静置, 沸水浴中加热 $2 \mathrm{~min}, 24500 \times g$ 离心 10 min. 所得上清液,即是 AAS 待测样品.

（2）AAS 测定. 所用原子吸收光谱仪为日立 180-80 型,用石墨炉(无火焰)测定 Cd, 塞 曼扣除背景, 原子化温度为 $1800^{\circ} \mathrm{C}$, 以提取液调空白. 数据处理模式采用峰面积计算方法, 所 得 $\mathrm{Cd}$ 含量测定数据单位为 $\mathrm{ppb}$. 根据 MTs 分子量以及每个 MTs 分子所结合的 Cd 原

1991-11-01 收稿, 1992-03-06 收修改稿

* 国家自然科学基金资助项目 
子数, 就可计算出每克肝脏中 $M T s$ 含量, 即 $M T s \mu g / g$ 肝组织.

3. MT-I 和 MT-II 分离制备 按照 Tsunoo 和 Kino 等人 ${ }^{[2]}$ 的方法.

4. MTs 的诱导 取 $20,35,180,240$ 日龄的小鼠各 20 只,分成两组. 每组均用以生理 盐水配制的 $\mathrm{CdCl}_{2}$ 溶液进行皮下注射, 共 5 次. 第 $\mathrm{I}$ 组注射标准是: 第 1,3 天 $1.5 \mathrm{mg} / \mathrm{kg}$ 体: 重; 第 5,7 天 $2.5 \mathrm{mg} / \mathrm{kg}$ 体重; 第 9 天 $4.5 \mathrm{mg} / \mathrm{kg}$ 体重. 第 II 组注射标准是: 第 1 ， 3 天 2.5 . $\mathrm{mg} / \mathrm{kg}$ 体重; 第 5,7 天 $4.0 \mathrm{mg} / \mathrm{kg}$ 体重; 第 9 天 $7.5 \mathrm{mg} / \mathrm{kg}$ 体重. 第 10 天断头处死小 鼠, 取 出肝脏, 按上述方法制备 AAS 待测样品.

5. 消除 $\cdot \mathrm{OH}$ 自由基能力的测定 主要参考王成莲和刘莉 ${ }^{[3]}$ 的方法. 反应体系中, 抗 坏血酸 $100 \mu \mathrm{mol} / \mathrm{L}, \mathrm{CuSO}_{4} 100 \mu \mathrm{mol} / \mathrm{L}$, 水杨酸 $2 \mathrm{mmol} / \mathrm{L}$, 细胞色素 $\mathrm{c} 100 \mu \mathrm{mol} / \mathrm{L}$, 磷酸二 氢钾缓冲液 $0.15 \mathrm{~mol} / \mathrm{L}, \mathrm{pH} 7.4, \mathrm{MTs}$ 粗提取液 $0.3 \mathrm{ml}$. 最终反应体积为 $2 \mathrm{ml}, 25^{\circ} \mathrm{C}$ 温 育 ${ }^{9} 0$ $\min$, 在 $550 \mathrm{~nm}$ 波长下测定其光密度值. 对照组以磷酸二氢钾缓冲液取代 MTs 粗提取液, 其他成分和浓度同样品组.

\section{6. 蛋白质含量测定 参照 Bradford 和 Sedmak 等人 ${ }^{[4,5]}$ 的方法.}

\section{二、实 验 结 果}

1. 小鼠肝脏 MTs 含量水平随日龄变化表 1 所列数据表明, ICR/JCL. 雄性小鼠肝 赃 MTs 含量水平呈现随日龄提高的趋势. 与 20 日龄小鼠肝脏 MTs 含量比较, 除 35 日龄小 鼠肝脏 MTs 含量变化不显著外, 180 日龄和 240 日龄小鼠肝脏 MTs 含量增加了 $30 \%$ 左右, 而 420 日龄小鼠肝脏 MTs 含量竟增加了近 $60 \%$. 由此可以看出, MTs 含量水平随日龄变 化是很显著的. 表 1 中数值是 2 次独立试验的平均值, 每次诖验有 3 个重复. 以下几个表中 的数值也是这样取得的.

表 1 小鼠肝脏 MTs 含量随日龄变化趙势

\begin{tabular}{c|c|c|c|c|c}
\hline \multicolumn{1}{c|}{$\begin{array}{c}\text { 日龄 } \\
\text { 含最 }\end{array}$} & 20 & 35 & 180 & 240 & 420 \\
\hline $\begin{array}{c}M T s(\mu \mathrm{g} / \mathrm{g} \text { 肝组织 }) \\
(\bar{x} \pm s \bar{x})\end{array}$ & $\begin{array}{c}21.02 \pm 0.42 \\
(n=10)\end{array}$ & $\begin{array}{c}22.32 \pm 1.17^{\mathrm{a})} \\
(n=10)\end{array}$ & $\begin{array}{c}28.06 \pm 0.48^{\mathrm{b})} \\
(n=10)\end{array}$ & $\begin{array}{c}28.28 \pm 0.46^{\mathrm{b})} \\
(n=10)\end{array}$ & $\begin{array}{c}\left.33.48 \pm 0.91^{\mathrm{b}}\right) \\
(n=10)\end{array}$ \\
\hline
\end{tabular}

a) $P>0.05$, b) $P<0.01$

表 2 不同剂量 $\mathrm{CdCl}_{2}$ 诱导不同日龄小鼠时其肝脏 $\mathrm{MTs}$ 含量的变化

\begin{tabular}{|c|c|c|c|c|c|}
\hline \multicolumn{2}{|l|}{ 含是 } & 20 & 35 & 180 & 240 \\
\hline MTs ( $\mu \mathrm{g} / \mathrm{g}$ 肝组织) & 组 & $\begin{array}{c}46.58 \pm 0.59 \\
(n=9)\end{array}$ & $\begin{array}{c}47.02 \pm 0.39 \\
(n=9)\end{array}$ & $\begin{array}{c}47.34 \pm 0.73^{81} \\
(n=8)\end{array}$ & $\begin{array}{c}\left.47.56 \pm 0.47^{2}\right) \\
(n=8)\end{array}$ \\
\hline$(\bar{x} \pm s \bar{x})$ & $\begin{array}{l}\text { I1 } \\
\text { 组 }\end{array}$ & $\begin{array}{c}60.45 \pm 0.69 \\
(n=9)\end{array}$ & $\begin{array}{c}61.32 \pm 0.44 \\
(n=8)\end{array}$ & $\begin{array}{c}\left.61.75 \pm 0.42^{3}\right) \\
(n=8)\end{array}$ & $\begin{array}{c}\left.62.07 \pm 0.53^{3}\right) \\
(n=7)\end{array}$ \\
\hline
\end{tabular}

a) $P>0.05$

2. 用相同剂量 $\mathrm{CdCl}_{2}$ 诱导不同日龄小鼠, 其肝脏 $\mathrm{MTs}$ 含量的变化 大量研究表明, 动物组织中 MTs 含量变化是与诱导物剂量有关. 在本研究中, 我们也观察到了这种相关 
倠. 从表 2 中的数据可以看出, 当用不同剂量的 $\mathrm{CdCl}_{2}$ 诱导 $\mathrm{I} 、 \mathrm{II}$ 两组不同龄小鼠时, 同组内? 不同日龄小鼠肝脏中 MTs 含量水平变化不显著, 但是 I、II 两组同龄小鼠之间相比却想差 近 $30 \%$. 即小鼠肝脏 MTs 含量水平, 只与诱导物剂量有关, 而与小鼠日龄关系不大.

3. MTs 消除 $\cdot \mathrm{OH}$ 自由基能力的变化动态表 3 数据表明, 小鼠肝脏 $\mathrm{MTs}$ 消除 $\cdot \mathrm{OH}$ 自由基能力随日龄呈上升趋势. 与 25 日龄小鼠肝脏 $\mathrm{MTs}$ 消除 ${ }^{*} \mathrm{OH}$ 自由基能力相比较, 56 . 日龄差异不显著, 180 日龄差异显著, 270 日龄差异就极为显著. 这种动态趋势, 与 MTs 含量 水平随日跉增加呈正相关性. 这暗示很可能是由于 $\mathrm{MT}$ s 量的增加, 才导致 $\mathrm{MTs}$ 消除 ${ }^{\circ} \mathrm{OH}$ 自. 由基能力的提高.

4. MTs 两种亚型消除 ${ }^{\circ} \mathrm{OH}$ 自由基能力的比较 MTs 有 $\mathrm{MT}-\mathrm{I}$ 和 MT-II 两种亚 型. 表 4 中的数据表明，这 2 种亚型消除 ${ }^{\circ} \mathrm{OH}$ 自由基的能力有显著的差异. MT-II 消除 $\cdot \mathrm{OH}$ 自由基的能力远远大于 $\mathrm{MT}-\mathrm{I}$, 前者是后者的 2 倍以上. 看来这两种亚型的生理功能. 很可能是不一样的.

表 3 不同日龄小鼠肝脏 $\mathrm{MTs}$ 消除 $\cdot \mathrm{OH}$ 自由基能力比较

\begin{tabular}{c|c|c|c|c}
\hline \multicolumn{1}{c|}{ 日舲 } & 25 & 56 & 180 & 270 \\
\hline $\begin{array}{c}\Delta A / \mathrm{mg} \text { 蛋白 } \\
(\bar{x} \pm s \bar{x})\end{array}$ & $\begin{array}{c}0.250 \pm 0.021 \\
(n=10)\end{array}$ & $\begin{array}{c}\left.0.259 \pm 0.029^{\mathrm{a}}\right) \\
(n=10)\end{array}$ & $\begin{array}{c}0.289 \pm 0.018^{\mathrm{b})} \\
(n=10)\end{array}$ & $\begin{array}{c}\left.0.312 \pm 0.038^{c}\right) \\
(n=10)\end{array}$ \\
\hline
\end{tabular}

a) $P>0.05$, b) $0.01<P<0.05$, c) $P<0.01$

* $\Delta A / \mathrm{mg}$ 蛋白为每撉克样品蛋白所增加的光密度读数

表 4 MTs 2 种亚型消除 $\cdot \mathrm{OH}$ 自由基能力的比较

\begin{tabular}{c|c|c}
\hline 亚型 & $\Delta A / \mathrm{mg}$ 䖪白 $(\bar{x} \pm s \bar{x})$ & $\frac{M T-I I}{\mathrm{MT}-\mathrm{I}}$ \\
\hline MT-I & $0.306 \pm 0.031$ & $2.24($ 倍 $)$ \\
MT-II & $0.685 \pm 0.048$ & \\
\hline
\end{tabular}

\section{三、讨 论}

我们曾用比较灵敏的极谱氧电极法,在相同的条件下,进行 SOD 与 $M T s$ 歧化 $\mathrm{O}_{i}^{-}$自由 基能力的比较, 发现后者仅是前者的几十分之一, 即 $\mathrm{MTs}$ 消除 $\mathrm{O}_{i}^{-}$自由基能力远不如 SOD, 与 Thornally 和 Vasak 的报道 ${ }^{[1]}$ 相一致.

MTs 是一种可诱导的蛋白质,它的含量与诱导物的剂量有关. 我们所观察到的小鼠肝脏 MTs 含量水平呈随日龄增加的趋势, 很可能是由于小鼠体内积累的 MTs 诱导物随日龄逐渐 增多所致. 至今已有不少论文都已证实, 在衰老过程中 SOD 比活总的趋势是随日龄降低, 过 氧化脂质的含量却是随日龄增加. 同时实验也䘚明, MTs 在细胞中有抑制脂质过氧化反应 的作用 ${ }^{[6-8]}$. 由此看来, MTs、SOD 和谷胱甘肽等很可能是共同调节着自由基的产生和消除, 使之处于一种动态平衡状态. MTs 和 SOD 基因的表达, 有可能是受到某种物质的共同调 节.

第 20 期 
实验结果不但证实了小鼠肝脏中 MTs 对消除 ${ }^{\circ} \mathrm{OH}$ 自由基是十分有效的,而且还首次证 明小鼠肝脏 MTs 消除" $\mathrm{OH}$ 自由基的能力是随日龄变化的. 由于 MTs 可猝灭对细胞毒性 极大的“ $\mathrm{OH}$ 自由基, 是细胞防御体系中一个重要成员, 因此我们认为 MTs 本身可能就是一 种含金属的蛋白酶; MTs 可能在延缓机体衰老过程中起着重要的作用.

本研究还首次提供了 MTs 2 种亚型具有不同的消除 ${ }^{\circ} \mathrm{OH}$ 自由基能力的实验证据. MTII 消除 ${ }^{\circ} \mathrm{OH}$ 自由基的能力是 $M T-I$ 的 2 倍以上,它提示 $M T s 2$ 种亚型可能担负着各自不同 的生理功能, 如 MT-I 主要是参与重金属的解毒作用等, 而 MT-II 可能在防御生物膜和染色 体免受 ${ }^{\circ} \mathrm{OH}$ 自由基损伤、维护细胞或机体的正常功能等方面起到重要的作用. 潘爱华等人在 研究辐射诱导 MTs 产生的实验中发现, MT-II 的含量增加 40 倍左右时, MT-I 仅增加 10 倍左右, 两者相差约 4 倍”. 实验已证明 MTs 含量水平呈随日龄增加趋势, 并且 MT-II 量又 多于 MT-I, MT-II 消除" OH 自由基能力又比 MT-I 大, 因此小鼠肝脏中 MTs 消除 ${ }^{\circ} \mathrm{OH}$ 自由基能力显然会呈现出随日龄上升趋势.

\section{参考文献}

[1] Hhornally, P. J., Vasak, M., Biochim. Biophys. Acza, 827(1985), 36.

[2] Tsunoo, H., Kino, K. et' al., J. Biol. Chem., 253(1978), 4172.

[ 3 ] 王成莲、刘莉, 生物化学与生物物理进展, 16(1989), 473-475.

[4] Bradford, M. M., Anal. Biochem., 72(1976), 248-254.

[ 5 ] Sedmak, J, J., Grossberg, S. E., Anal. Biochem., 79(1977), 544-552.

[6] Slater, T. F., Ciba. Found. Symp., 65(1979), 143-151.

[ 7 ] Menzel, D. B., Annu. Rev. Pharmacol., 10(1979), 375-394.

[8] Brigelius, R., Spottl, R. et al., FEBS Lett, 47(1974), 72-75.

1) 潘爱华等,已投中华放射医学及防护杂志 